Conclusion YF476 is a promising new medical treatment for type I gastric neuroendocrine tumours. It appears to be well tolerated with no observed toxicity. Further trials of YF476 involving more prolonged treatment regimes are therefore warranted in this condition.

Competing interests A Moore grant/research support from: Trio Medicines Ltd., L Ball: None declared, M Boyce: None declared, A Varro: None declared, D Pritchard: None declared.

\section{OC-098 HOW COMMONLY IS GASTRIC CANCER MISSED AT ENDOSCOPY: A UK PRIMARY CARE BASED STUDY}

doi:10.1136/gutjnl-2012-302514a.98

${ }^{1} \mathrm{~S}$ Menon, ${ }^{*}{ }^{2} \mathrm{~A}$ Dhar, ${ }^{3} \mathrm{~J}$ Hoare, ${ }^{4} \mathrm{~N}$ Trudgill. ${ }^{1}$ Department of Gastroenterology, New Cross Hospital, Wolverhampton, UK; ${ }^{2}$ Department of Gastroenterology, Bishop Auckland Hospital and Darlington Memorial Hospital, Darlington, UK; ${ }^{3}$ Department of Gastroenterology, St.Mary's Hospital, London, UK; ${ }^{4}$ Department of Gastroenterology, Sandwell General Hospital, West Bromwich, UK

Introduction Meta-analysis of published single hospital series including 1977 subjects suggests that 14\% of gastric cancer (GC) subjects have had an upper gastrointestinal endoscopy (OGD) up to 3 years previously that failed to diagnose their GC $(50 \%$ in the 12 months before diagnosis and 50\% $1-3$ years before diagnosis).

Methods All patients with GC in the THIN general practice database covering 5 million UK subjects were examined. A nested casecontrol study was performed with cases subjects who underwent OGD 1-5 years prior to their OGD that diagnosed GC and controls subjects who did not undergo OGD $1-5$ years prior to their diagnostic OGD.

Results 5473 GC were available for analysis (3402 males (62\%), mean age 71 years), with follow-up of 46779 subject years. 169 (3.1\%, 98 males (58\%), mean age 71 years) had an OGD which failed to diagnose GC between 1 and 5 years prior to diagnosis of GC, out of whom $128(2.3 \%)$ had OGD between 1 and 3 years before and 41 $(0.7 \%)$ had an OGD between 3 and 5 years before diagnosis. There were 56 primary care consultations with symptoms pertaining to oesophago-gastric cancer between 1 and 5 years prior to diagnosis of GC (dyspepsia (n) 51, anaemia 9, weight loss 12, dysphagia 3), of which all underwent OGD. No subject who had an OGD that did not diagnose cancer was on proton pump inhibitor (PPI) therapy in the year prior to OGD and $49(0.9 \%)$ of subjects were on PPI therapy in the year prior to being diagnosed with GC. Logistic regression analysis of subjects who had an OGD that failed to diagnose cancer and those that did not, failed to identify any specific predictive factors (age 1.0 (0.99-1.01), $\mathrm{p}=0.99)$, sex 0.84 (0.61-1.14), $\mathrm{p}=0.26$ ), related to an OGD that failed to diagnose GC.

Conclusion Missing GC at OGD is, reassuringly, less than half as common as previous much smaller studies in secondary care have suggested. PPI therapy does not contribute to missing GC at OGD. Advances in endoscopy and selection bias in previous studies may account for these differences.

Competing interests None declared.

\section{OC-099 INCREASED AT RISK SCREENING AND RECOGNITION OF ATYPICAL PRESENTATION DOES NOT FULLY EXPLAIN THE 6.5-FOLD INCREASE IN PAEDIATRIC COELIAC DISEASE (CD) INCIDENCE IN THE LAST 20 YEARS IN SE SCOTLAND}

doi:10.1136/gutjnl-2012-302514a.99

${ }^{1} V$ Merrick, ${ }^{*}{ }^{2} L$ White, ${ }^{2} E$ Bannerman, ${ }^{1} R$ K Russell, ${ }^{1} D$ Basude, ${ }^{1} \mathrm{P}$ Henderson, ${ }^{3} \mathrm{D}$ C Wilson, ${ }^{1} \mathrm{P}$ M Gillett. ${ }^{1}$ Paediatric Gastroenterology and Nutrition, Royal Hospital for
Sick Children, UK; ${ }^{2}$ Dietetics, Nutrition and Biological Health Sciences, Queen Margaret University, UK; ${ }^{3}$ Child Life and Health, University of Edinburgh, Edinburgh, UK

Introduction Current diagnostic practice for paediatric CD in the UK includes increased screening of family members and at- risk groups for example, diabetes mellitus.

Aims To identify all incident cases of CD in SE Scotland over the 20year period of 1990-2009 to assess trends in incidence, symptomatology, age at diagnosis and the impact of active screening of atrisk groups. Utility of routine laboratory tests at diagnosis was also evaluated (2005-2009 diagnoses only).

Methods A retrospective review of case notes, pathology databases, endoscopy and patient records was performed for all children diagnosed with CD <16 years from 1990 to 2009 on duodenal biopsy in SE Scotland (at-risk population group of 233000 aged <16 years). Data were age-sex standardised and analysed in 5-year epochs, with Poisson regression models used to calculate changes in incidence over time.

Results 266 biopsy positive children were diagnosed from 1990 to 2009 with an increase in incidence from 1.8 (95\% CI 1.1 to 2.7$)$ to 11.7 (95\% CI 9.8 to 13.9 ) per 100000 children aged <16 years during the 1990-1994 and 2005-2009 epochs respectively ( $p=0.001)$. The median age (IOR) at diagnosis also increased significantly from 29 (16-53) months to $90(53-132)$ between these epochs $(p<0.0001)$, and non-classical presentation (children with a mono-symptomatic presentation and those with extra-intestinal symptoms for example, fatigue, pallor, irritability) increased significantly from 5\% to $21 \%$ respectively $(p=0.008)$. Additionally, $7 \%$ of children were diagnosed through targeted screening in 1990-1994 compared to $23 \%$ in 2005-2009 ( $p=0.002)$. When cases identified via active screening or with non-classical symptoms were removed, a significant rise from 1.51 (95\% CI 0.91 to 2.38 ) in $1990-1994$ to 6.59 (95\% CI 5.17 to $8.28)$ in 2005-2009 ( $p=0.006)$ remained. Routine blood investigations demonstrated that over $25 \%$ were anaemic and over $50 \%$ were iron deficient at presentation in 2005-2009.

Conclusion The incidence of paediatric $\mathrm{CD}$ has increased 6.5-fold over the last 20 years in SE Scotland. Children are older at diagnosis, presenting with fewer classical and more varied symptoms, and with iron deficiency anaemia the commonest laboratory abnormality. We show that both increased screening of at-risk groups and a lower threshold for serological screening (as a result of greater clinician awareness of the condition's heterogeneic nature) do not fully explain the overall rise in incidence.

Competing interests None declared.

\section{OC-100 THE CLONAL ORIGINS OF GASTRIC ADENOCARCINOMA}

doi:10.1136/gutjnl-2012-302514a.100

${ }^{1} \mathrm{~T}$ Ventayol Garcia, ${ }^{*} \mathrm{M}$ Rodriguez-Justo, ${ }^{3} \mathrm{~T}$ Graham, ${ }^{2} \mathrm{M}$ Novelli, ${ }^{1} \mathrm{~N}$ Wright, ${ }^{1} \mathrm{~S}$ McDonald. 'Deparment of Digestive Diseases, Blizard Institute, Barts and the London school of Medicine and Dentistry, OMUL, UK; ${ }^{2}$ Deparment of Histopathology, University College London, London, UK; ${ }^{3}$ Deparment of Histopathology, Cancer Research UK, London, UK

Introduction We have previously shown that entire fields of dysplasia in the human stomach are derived from a single mutated, metaplastic gland. ${ }^{1}$ This suggests that intestinal metaplasia (IM) can be considered a field defect among which dysplasia can arise and this would indicate that adenocarcinomas derived from such dysplasia would also be clonal. Recent work published by our laboratory has indicated that familial adenomatous polyposis-associated colorectal adenomas as well as some sporadic lesions ${ }^{2}$ and dysplasia within Barrett's oesophagus ${ }^{3}$ are polyclonal. There is therefore a need to ascertain the clonality of gastric adenocarcinomas (GA). 